\title{
Exploring Cinema Databases using multi-dimensional Image Measures
}

\author{
Robin G. C. Maack*, David H. Rogers ${ }^{\dagger}$, Hans Hagen*, Christina Gillmann* \\ ${ }^{*}$ Computer Graphics and HCI Lab, University of Kaiserslautern, Kaiserslautern, Germany \\ E-mail: maack@rhrk.uni-kl.de, hagen@cs.uni-kl.de, c_gillma@cs.uni-kl.de \\ ${ }^{\dagger}$ Data Science at Scale, Los Alamos National Laboratory, Los Alamos, USA \\ E-mail: dhr@lanl.gov
}

\begin{abstract}
Exa-scale simulations can be hard to analyze because it is nearly impossible to store all computed time-steps and other parameters. The Cinema Database provides a storagesaving solution, that captures images of each simulation time-step from a variety of camera angles. Still, the resulting number of images can be overwhelming and it is hard to find interesting images and features for further analysis. We present a zoom based approach where users can utilize arbitrary image measures to explore interesting images and further analyze their behaviour in detail. We showed the effectiveness of our approach by providing two real world Cinema datasets.

Index Terms-Cinema Database, Image Database, Image Measures, Data compression, Visualization, Data Exploration
\end{abstract}

\section{INTRODUCTION}

Large image databases recently have been used more frequently, either as commercial tools such as Bynder ${ }^{1}$ or pixx.io ${ }^{2}$ as well as in research such as the Cinema Database ${ }^{3}$ [1], [2] and interactive websites ${ }^{4}$. Their general purpose is to allow users to browse, select and analyze large collections of images. Cinema in particular aims to assist in the analysis of exascale simulations, with multiple time-steps that are too big to be stored on a computer or cluster. It consists of multiple projects that can create Cinema Databases from simulations, alter and add additional information to Cinema Databases, visualize those databases in viewers, as well as providing building blocks to build up a new viewer.

Here, Cinema provides a variety of images per time-step that can be stored and reviewed. This enables the user to watch the simulation as often as needed to analyze problems and to find interesting results, while not requiring a lot of compute power or massive storage solutions. Although Cinema Database assists users in storing, managing and browsing their image collection, it lacks the ability to provide an overview of the image collection, an entry point for image searches or a search function via interesting attributes. As one can imagine, not every image in the Cinema Database provides the same amount of information. Usually, time-steps are sampled equidistant in each direction leading to a variety of images

LEVIA'19: Leipzig Symposium on Visualization in Applications 2019

${ }^{1}$ https://software-info.gartnerdigitalmarkets.com/bynder

${ }^{2}$ https://www.pixxio-bildverwaltung.de

${ }^{3}$ https://cinemascience.github.io

${ }^{4}$ http://johnguerra.co that can show equivalent or irrelevant information. When considering these image collections, users face the problem of finding interesting images or overseeing interesting ones for their analysis as shown in Section II.

To solve this problem, this paper provides a novel visual guidance approach that can be used to explore large image databases. It is based on a zoom approach. We provide a visual workflow embedded in the Cinema Database where users can determine arbitrary image metrics that capture features of interest. In most cases, multiple features are of interest to the user and the determination of their importance is part of the exploration process. To address this issue we allow to implement multiple image measures and provide users with a visual tool to explore these metrics, apply aggregation functions and explore the resulting selection based on the user settings. When focusing on selected images we provide a detailed view of the selected features within the images.

This paper contributes:

- Requirements for intuitive exploration of large image databases

- A context view of feature distribution within a Cinema Database

- A detail view of feature distribution within images

We show the effectiveness of the presented approach by applying it to two different Cinema Databases (see Section IV).

\section{RELATED WORK}

This Section summarizes the state of the art for the visualization and exploration of large image databases.

\section{A. Visualization of Large image databases}

As stated by Camargo et al. [3], most image databases aim to map a high-dimensional feature space into a twodimensional map. This allows to visually express closeness of images. A variety of approaches are available, namely Multidimensional Scaling [4], Principal Component Analysis [5], Self-Organizing Maps [6], Curvilinear Component Analysis [7], Laplacian Eigenmaps [8], Shannon's Mapping [9], Stochastic Neighbor Embedding [10] or Kernel Isomaps [11].

Although these approaches allow users to gain a first overview over the considered image database, they lack the 


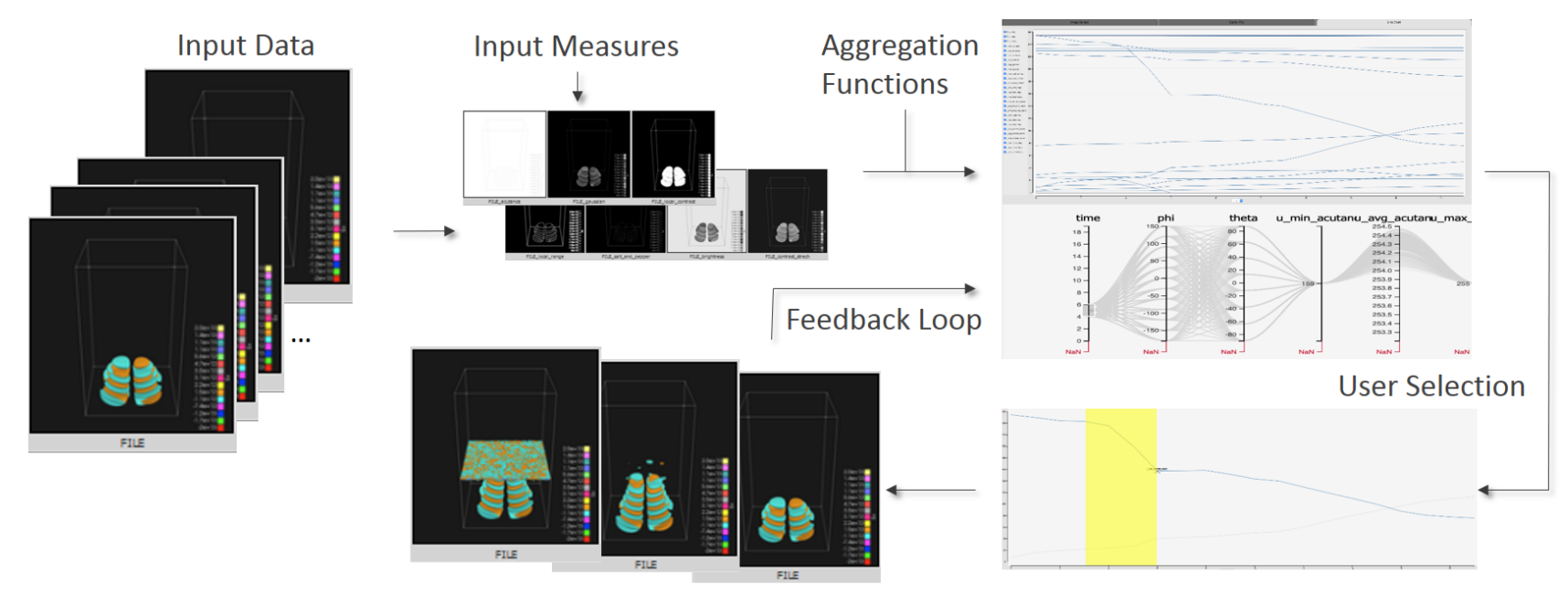

Fig. 1. Zoom based Querying in Cinema Database. Input images, input measures and aggregation functions form the input of the presented approach. Users can select interesting time-steps, where image features change dramatically. The selected images can be reviewed and form a feedback loop for users in order to create new selections.

ability to guide users to interesting images, that contain most of the information. The presented approach allows users to select different features of images and identify images, that match these features the most.

\section{B. Exploration of Large image databases}

There has been a large number of works addressing the navigation of large image collections. The most important will be discussed in the following.

Deng et al. [12] presented a similarity based visualization of large image collections, that should allow users to identify images that contain similar content. Although this allows users to identify similar images, it does not provide an entry point, where users are guided to interesting images. In contrast to this, we provide a measure-based entry point where users can identify interesting images and further examine them.

Further approaches utilize hierarchical visualizations of images to allow users to identify correlations between the reviewed images [13]-[15]. Here, images are ordered in terms of their hierarchical correlation, which can be computed with different measures. Although this provides an important information about the hierarchy within an image database, users need to be assisted in finding the most significant images. In contrast to the presented approaches, we provide a zoom based approach where users can select interesting images and allow further examination.

Image databases can additionally be browsed and sorted according to meta data information, such as segmentation information [16], machine-learning based content examination [17], [18], Thumbnails [19] and image locations and time [20], [21]. Here, users are enabled to search for interesting images as a starting point. Still, the meta-data needs to be computed, which can be computationally expensive. In the presented system we provide a measure-based approach, where users can insert and pre-compute arbitrary measures to query interesting images in the considered image database.

\section{Requirements Analysis}

In order to develop an interactive framework where users can find and examine interesting images in a large image collection, we formulate requirements based on the conducted state of the art analysis that needs to be fulfilled.

a) R1: Feature Selection: The selection of images in a database is highly depending on the goal of the user. Here, users need to be enabled to determine features they are interested in and utilize these feature measures to select interesting images.

b) R2: Overview of Feature Distribution: Besides the ability to freely decide on image features, users need to be able to browse the overall distribution of a feature in the considered image collection. Solely when reviewing the overall distribution of a feature in a set of images, they are able to recognize important images where specific features are dominant.

c) R3: Detail view of Interesting Images: Although the selected images may represent a suitable set of images that contain the desired feature, users still need to be able to review single images and explore how features are distributed in these images.

d) R4: Low computational and usage costs: As the Cinema Database is designed to decrease the computational costs of reviewing simulation results, a navigation approach should not increase the complexity of the Cinema approach [22].

\section{METHODS}

This section describes the presented visual guidance in Cinema databases using multi-dimensional image measures. An overview over the workflow we present is shown in Figure 1. Starting from the input dataset and the selected image measures, aggregation functions are used to interactively guide users to interesting images in the database. Followed by that users can further analyze selected images in detail by 
reviewing all selected image features in detail. The single steps will be shown in the following.

\section{A. Input and Preprocessing}

Input datasets that fit the Cinema Database scheme can be very versatile. The current specification $\left(\operatorname{spec} \mathrm{D}^{5}\right)$ requires a cvs file containing all images and additional information that is contained in the database. It can state additional metadata for example angle positions of the camera as well as time-step information and even additional data files.

In addition to the input Cinema Database, the presented approach requires one or multiple image measures, that represent the features of interest in the given database. These features can range from image quality measures [23] over uncertainty measures [24] to entropy measures [25]. Computing image measures can be time-consuming especially when considering large image databases. As these computational outputs remain the same, we pre-compute the selected image measures and store them beside the actual images. When starting the presented system, the computed measures can be directly loaded and no additional time is required.

The used image measures vary in their goals, but are all local to a pixel rather than the whole image. To give a short overview, all used measures will be briefly described. Acutance [26] is an image measure which considers pixels on an edge as certain resulting in a low output value for those pixels. Contrast Stretch [27] on the other hand aims to have an equal distribution of intensity values, giving pixels with very low or very high occurrence rates a high value. Gaussian Noise [28] is very similar, but uses a gaussian distribution instead. Brightness [29] is a simplistic measure which considers dark pixels as certain and gives them a low value. Local Contrast [30] views pixels which are often used as trustworthy resulting in a low value. Local Range [31] uses the highest absolute distance to its neighbors as the output. And finally, Salt and Pepper [32] uses the average distance to the neighboring pixels for its output.

Usually image measures work on a local level, which means that they output a result for each pixel contained in an image. As we are interested in the overall rating of an image in order to find interesting ones, we need to accumulate the single outputs of each pixel in an image. Here, the presented system utilizes different aggregation functions, such as minimum, maximum or average, to create an overall impression of single images. An overview of different aggregation functions can be seen in the work of Cai et al. [33].

In summary, the image collection, feature measures and aggregation functions form the input of the presented system.

\section{B. Feature Aggregation Visualization}

Based on the pre-processed input of the presented system, we built an interactive visualization where users can search for interesting images in the database and further examine them. Here, we first need to provide an intuitive visualization to allow users to find interesting images along different

\footnotetext{
${ }^{5}$ https://github.com/cinemascience/cinema/tree/master/specs/dietrich
}

computational time-steps of a simulation. The presented visualization consists of multiple components. First, a line-chart that represents the accumulated feature functions across the considered time-steps or other dimensions. Second, a parallel coordinates view that represents the distribution of aggregated features throughout the captured images. Third, an image viewer showing each image with it's corresponding image measure images. The first two views can be seen in Figure 2.

Figure 2 a) shows the line chart of the aggregated feature functions. The $\mathrm{x}$-axis presents the selected dimension, here the time-steps are shown, and the y-axis shows the aggregated feature values. Users can enable and disable all feature functions and search for interesting trends of one or multiple features. When detecting interesting feature trends at specific time-steps, users can select these time-steps by dragging along the $\mathrm{x}$-axis and the corresponding images will be displayed.

Figure $2 \mathrm{~b}$ ) represents an example of the parallel coordinate view. Here axis like time and angles of the captured simulation runs and the computed aggregation functions are displayed. Users can manipulate the view by sorting the axis as well as selecting interesting feature accumulations or time-steps. The selected images will be displayed according to the user selection.

Both views allow users to examine the image database according to the defined image feature functions and the selected aggregation functions. The selections made throughout this interaction process will be displayed to the user and can be further examined in detailed view.

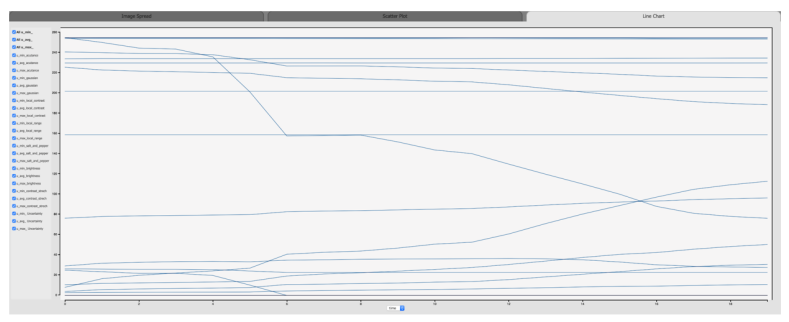

a)

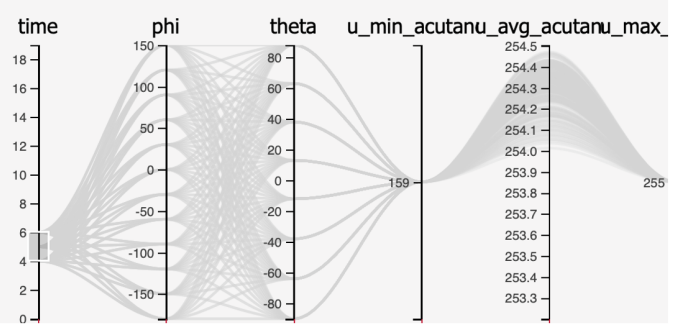

b)

Fig. 2. Feature aggregation visualization. a) Line plot with $x$-axis showing the time-step and y-axis representing several aggregated feature values. b) Parallel coordinates plot with aggregated feature values, time and image angles.

\section{Image Features Visualization}

After selecting interesting images based on the aggregated measures, users are enabled to review the selected images and 
their features. Contrary to the aggregated views, we aim to allow users to examine features of the selected images.

Here, we present all computed features side by side. Users can review each output of the computed measures in order to determine areas in the image that contributed most to the aggregation function output. Although not available yet, a further visualization approach that helps users to review all measure outputs at once can be included in the presented system by utilizing an embedded isosurface visualization [34].

\section{Implementation}

For this project to be implemented, three repositories had to be altered. First, Cinema $\mathrm{Lib}^{6}$ is used to apply image measure functions, as well as aggregate functions to the images and save them to the database. Second, we added the Line Chart component to Cinema Components ${ }^{7}$. This Line Chart component is then embedded into Cinema Explorer ${ }^{8}$.

1) Cinema Lib: Cinema Lib is a command line tool written in Python for altering and checking Cinema databases. In our case, we implemented seven different image measures [26][32], [35] to the already existing image measures.

The chosen measures capture different properties of the images. For example, the Brightness measure [29] rates bright pixels as important and gives them a high value, where as dark pixels are considered insignificant. Other measures such as local range [31] and salt and pepper [32] work more like edge detectors together with smoothing operations, assessing pixels on sharp edges as significant.

These measures can be applied by a simple command that can be found in the Cinema Lib documentation. We also implemented a way to automatically apply the aggregate functions min, max and average to these resulting measure images, giving them a single value per time-step to be displayed.

Other image measures and aggregate functions can be added by expanding the functions in the Cinema Lib project. The functions for applying image measures to Cinema Databases already exist, so own implementations of image measures just have to be made compatible with these functions. Thus, it is rather easy to add new measurements to the system. The same holds for aggregate functions.

2) Cinema Components: Cinema Components is a collection of tools and visual components written in JavaScript. They are designed such that they can easily be embedded into any HTML viewer. We added a Line Chart component, as well as interaction methods for other components to this repository, enabling the user to explore the computed aggregate functions from the altered Cinema Database. The Line Chart component consists of the chart itself and a variety of user interface functions to create an easy to use component.

3) Cinema Explorer: Cinema explorer is an advanced viewer to explore Cinema Databases. It consists of a Parallel Coordinates view in the top and a tab container on the bottom.

\footnotetext{
${ }^{6}$ https://github.com/cinemascience/cinema_lib

${ }^{7}$ https://github.com/cinemascience/cinema_components

${ }^{8}$ https://github.com/cinemascience/cinema_explorer
}

The tab container has three tabs, an image viewer, a scatter plot and the new line chart.

The Parallel Coordinates (Fig. 2 b) view gives the user an overview over the dataset enabling the user to view parts of the data by selecting a range for each dimension.

The image viewer shows all images corresponding to an entry in the dataset, giving the user a detailed view of the dataset. Here all the image measures can be seen next to the original image, allowing the user to view all the details of the dataset together with the properties of each entry.

Scatter plots can be used to plot two dimensions against each other, to explore the interdependence of two dimensions.

The Line Chart shows the computed aggregate functions, averaged over the entries for the selected x-axis. For example, if the time-steps are selected, all those entries with the same time-step are averaged in regard to their aggregate function and then displayed. A resulting line might show that a certain measure is rising or falling on average, depending on the viewed measure. This can mean that the amount of edges, homogeneous regions or overall brightness of the images are rising of falling. The chart is embedded in a way that selections on the chart are also applied to the Image Viewer and the Parallel Coordinates view, granting the user easy control over the whole tool. Therefore, a part of the $\mathrm{x}$-axis can be selected on the Line Chart to be viewed in detail in the Image Viewer. This gives the user a zoom exploration experience.

4) General Workflow: The general workflow can be summarized to a few steps:

1) Convert a Cinema Database to support aggregated measurements of any kind using the Cinema Lib project.

2) Add the column names from the csv file of the aggregated measurements to the Cinema Explorer database file, using the image_measures entry.

3) Use the Line Charts checkboxes and filter for interesting looking or strongly changing lines, zooming in on them.

4) Select a part of the $x$-axis with interesting changes in the lines by dragging the mouse with a left click.

5) Find out what causes theses changes looking using the detailed information from the Image Viewer.

6) Repeat step 3-5 or 4-5 to fully explore the important steps in the dataset.

\section{RESUlts AND Discussion}

In this section we will present two successful Cinema Databases that can be reviewed by the presented workflow. Additionally, we will discuss the presented approach against the defined requirements.

\section{A. Results}

In order to prove the applicability of the presented system to real world datasets, this paper provides two use cases, that will be discussed in the following. For both use cases we provide the aggregation functions minimum, maximum and average as well as seven different image measures [34]. 


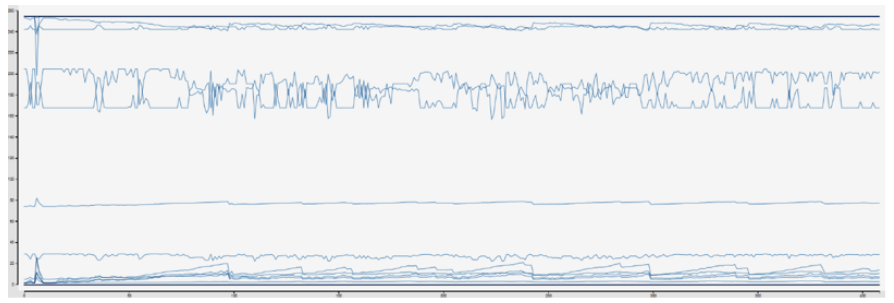

a)

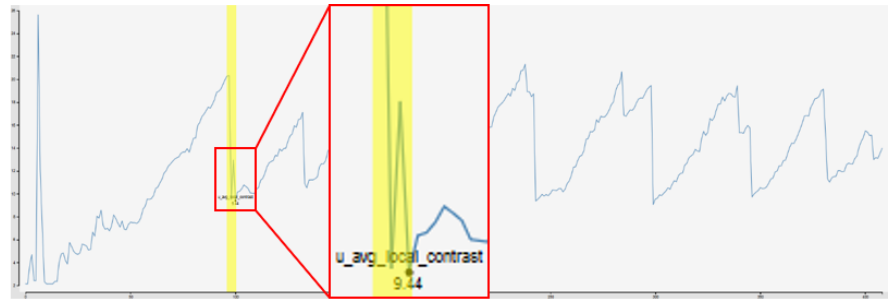

b)

Fig. 3. A snapshot of the Line Chart with the loaded Bubble Bed simulation. Fig. 3a shows all the available aggregate functions. In Fig. 3b a very characteristic function was selected, zooming into the range of that function. The yellow bar shows a selection of the user, which is viewed in detail in Fig. 4 . The zoomed part of the Line Chart shows a second rise and drop of the line after the huge drop.

Our first example is a series of time-steps comparing the evolution of isosurfaces based on topological analysis optimized to find the most significant isosurfaces versus regularly spaced isosurface values. The images are acquired by the WarpX system [36], which is an advanced particle accelerator simulation code that models plasma-based accelerators. The image database contains 1920 images in total. Scientists are interested in finding interesting time-steps where novel and large iso-surfaces occur, meaning that they are interested in big changes of the iso-surfaces. We use this example to show the resulting workflow of the presented approach, as can be seen in Figure 1.

When applying our algorithm, both aggregation function visualizations can be reviewed by the user. In the line aggregation view most values appear to be stable independently from the reviewed time-step. Contrary to this, maximum and minimum contrast stretch are increasing (or decreasing) when considering different time-steps. Here, users can select the most significant "jump" in the aggregation function. When reviewing the resulting images, it can be seen, that the images change dramatically. The shown iso-lines are massively growing until they finally build a novel large iso-surface. This example shows, that the presented system helps users in identifying interesting time-steps and review the content of these images.

1) Bubble Bed Simulation: The second example of a real world dataset is a small, dense bubbling bed with over $3 \mathrm{M}$ particles. The physical dimensions of the domain is about the size of a business card. This simulation contains 408 timesteps, $3 \mathrm{M}$ particles and displays the formation and evolution of bubbles expected in a full looping reactor. The data was generated by the MFIX-Exa system, which is a multiphase flow simulation code based on the MFIX code. Detailed information on the MFIX simulation can be found on the National Energy Technology Laboratory (NETL) website ${ }^{9}$.

To analyze this dataset, it is important to find time-steps that mark important steps in the simulation. Therefore seeing how much area is covered by bubbles at a time is a good insight to the scientist.

Fig. 3 a) shows all the available aggregate functions over all considered image measures. Now there are very characteristic

\footnotetext{
${ }^{9}$ https://mfix.netl.doe.gov/
}

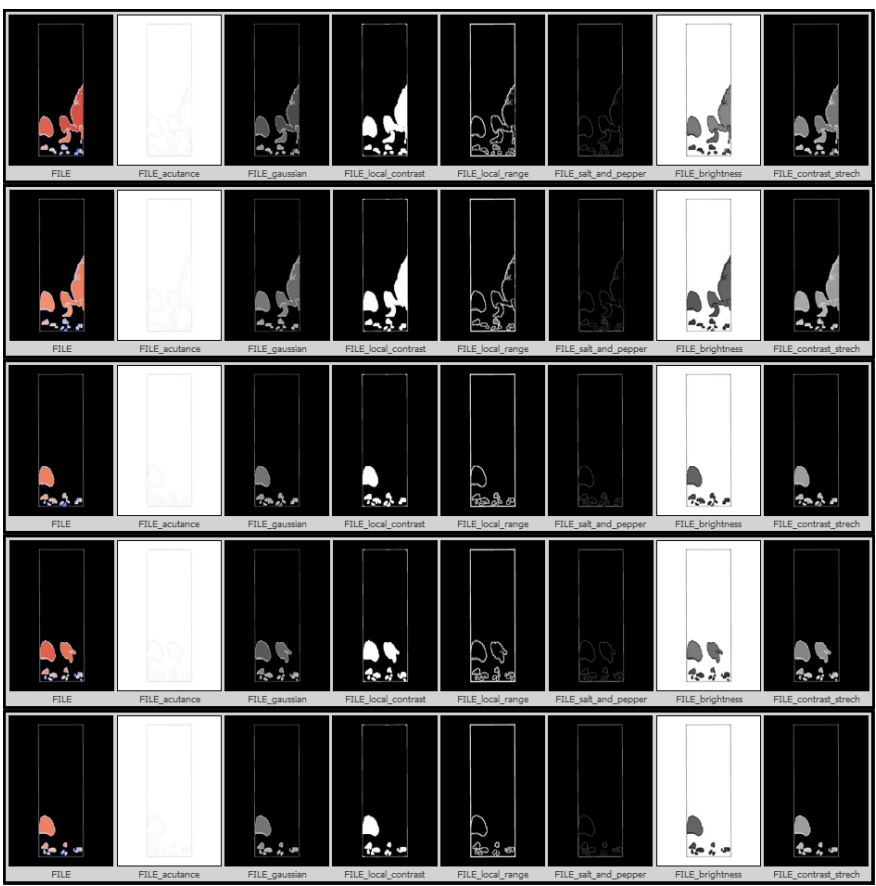

Fig. 4. A snapshot of the Image Viewer tab with the loaded Bubble Bed simulation showing the images selected in Fig. 3b. On the left the original images, each with 7 image metrics next to it, can be seen. Each row corresponds to one time-step. The sharp drop with the smaller spike afterwards in the selected region corresponds to a big bursting bubble followed by a smaller bubble building up and bursting again.

lines at the bottom of the chart that show a repetitive behaviour. When the top most line is selected, as seen in $3 \mathrm{~b}$ ), a line building up slowly and dropping very fast can be seen. Therefore a part of the $\mathrm{x}$-axis is selected(yellow) and is viewed in the Image Viewer tab in Fig. 4. When considering the selected images, the cause for the sharp drops can be found in the images, as these show how a big bubbles burst and grow over time. Bubbles building up and bursting according to the selected aggregated measurement can be seen. Now the scientist can characterize the dataset into growing stages and bursting stages by utilizing the selected image measure. 


\section{B. Discussion}

This section will discuss the defined requirements as well as the benefits and drawbacks of the presented approach.

The presented system is designed in order to allow a variety of image measures for the selection of interesting images. We implemented 7 different image measures available to users. Additionally, the code is designed flexible to allow users to further implement their own image measures (R1).

In order to provide a context visualization of all images contained in a Cinema Database, we allow the use of different aggregation functions and represent the results with two different line-views. In the current system, we implemented 3 different aggregation functions. Again, users are enabled to implement further aggregation functions due to the flexible code design (R2).

After deciding on interesting features, users are enabled to review selected images of the database. The images and computed measures can be reviewed for every single measure that was utilized for the selection process. This forms a feedback loop as users can check which part of the image contribution forms the outcome of the image measure (R3).

The presented system allows to add arbitrary image measures in order to classify images. Although these can be computationally expensive, the runtime of the presented workflow is not affected as the image measures are computed in a preprocessing step and loaded when required (R4).

Overall, the presented system is capable of fulfilling the defined requirements.

\section{COnClusion And Future Work}

We presented a flexible zoom based querying for image databases using multi-dimensional image measures. The system is designed to assist users in browsing large Cinema Databases in order to find interesting time-steps according to their own selected image measures. The system is designed flexible in order to enable users to insert novel image measures and aggregation functions. The effectiveness of our approach was demonstrated with two real world scenarios. As future work we are planning to provide multiple views for selected images where users can explore the composition of examined image measures within an image. Additionally, we are aiming to provide a similarity measure where users can directly find related images according to the selected image measures.

\section{REFERENCES}

[1] J. Ahrens, S. Jourdain, P. OLeary, J. Patchett, D. H. Rogers, and M. Petersen, "An image-based approach to extreme scale in situ visualization and analysis," in SC'14: Proceedings of the International Conference for High Performance Computing, Networking, Storage and Analysis. IEEE, 2014, pp. 424-434.

[2] S. C. Vogel, C. M. Biwer, D. H. Rogers, J. P. Ahrens, R. E. Hackenberg, D. Onken, and J. Zhang, "Interactive visualization of multi-data-set rietveld analyses using cinema: Debye-scherrer," Journal of applied crystallography, vol. 51, no. 3, pp. 943-951, 2018.

[3] J. Camargo, F. González, and R. Torres, "Visualization, summarization and exploration of large collections of images: State of the art," in LatinAmerican Conference On Networked and Electronic Media. LACNEM, 2009.
[4] J. Zhang, Visualization for information retrieval. Springer Science \& Business Media, 2007, vol. 23.

[5] S. T. Roweis and L. K. Saul, "Nonlinear dimensionality reduction by locally linear embedding," science, vol. 290, no. 5500, pp. 2323-2326, 2000.

[6] G. P. Nguyen and M. Worring, "Interactive access to large image collections using similarity-based visualization," Journal of Visual Languages \& Computing, vol. 19, no. 2, pp. 203-224, 2008.

[7] J. A. Lee and M. Verleysen, Nonlinear dimensionality reduction. Springer Science \& Business Media, 2007.

[8] J. B. Tenenbaum, V. De Silva, and J. C. Langford, "A global geometric framework for nonlinear dimensionality reduction," science, vol. 290, no. 5500, pp. 2319-2323, 2000.

[9] J. W. Sammon, "A nonlinear mapping for data structure analysis," IEEE Transactions on computers, vol. 100, no. 5, pp. 401-409, 1969.

[10] G. E. Hinton and S. T. Roweis, "Stochastic neighbor embedding," in Advances in neural information processing systems, 2003, pp. 857-864.

[11] H. Choi and S. Choi, "Robust kernel isomap," Pattern Recognition, vol. 40, no. 3, pp. 853-862, 2007.

[12] H. Liu, X. Xie, X. Tang, Z.-W. Li, and W.-Y. Ma, "Effective browsing of web image search results," in Proceedings of the 6th ACM SIGMM international workshop on Multimedia information retrieval, 2004, pp. 84-90.

[13] B. B. Bederson, B. Shneiderman, and M. Wattenberg, "Ordered and quantum treemaps: Making effective use of $2 \mathrm{~d}$ space to display hierarchies," AcM Transactions on Graphics (TOG), vol. 21, no. 4, pp. 833854, 2002.

[14] K. Andrews, W. Putz, and A. Nussbaumer, "The hierarchical visualisation system (hvs)," in 2007 11th International Conference Information Visualization (IV'07). IEEE, 2007, pp. 257-262.

[15] J. Deng, W. Dong, R. Socher, L.-J. Li, K. Li, and L. Fei-Fei, "Imagenet: A large-scale hierarchical image database," in 2009 IEEE conference on computer vision and pattern recognition. Ieee, 2009, pp. 248-255.

[16] W.-Y. Ma and B. S. Manjunath, "Netra: A toolbox for navigating large image databases," Multimedia systems, vol. 7, no. 3, pp. 184-198, 1999.

[17] A. Torralba, R. Fergus, and Y. Weiss, "Small codes and large image databases for recognition," in 2008 IEEE Conference on Computer Vision and Pattern Recognition. IEEE, 2008, pp. 1-8.

[18] R. Barber, W. Equitz, W. Flickner, W. Niblack, D. Petkovic, and P. Yanker, "Efficient query by image content for very large image databases," in Digest of Papers. COMPCON Spring. IEEE, 1993, pp. 17-19.

[19] P. Brivio, M. Tarini, and P. Cignoni, "Browsing large image datasets through voronoi diagrams," IEEE transactions on visualization and computer graphics, vol. 16, no. 6, pp. 1261-1270, 2010.

[20] A. Gomi and T. Itoh, "A personal photograph browser for life log analysis based on location, time, and person," in Proceedings of the 2011 ACM Symposium on Applied Computing. ACM, 2011, pp. 12451251.

[21] C. T. Vi, K. Takashima, H. Yokoyama, G. Liu, Y. Itoh, S. Subramanian, and Y. Kitamura, "D-flip: Dynamic and flexible interactive photoshow," in International Conference on Advances in Computer Entertainment Technology. Springer, 2013, pp. 415-427.

[22] C. Gillmann, H. Leitte, T. Wischgoll, and H. Hagen, "From Theory to Usage: Requirements for successful Visualizations in Applications," in IEEE Visualization Conference (VIS) - C4PGV Workshop, 2016.

[23] I. Avcibas, B. Sankur, and K. Sayood, "Statistical evaluation of image quality measures," J. Electronic Imaging, vol. 11, pp. 206-223, 2002.

[24] C. Gillmann, P. Arbeláez, J. T. Hernández, H. Hagen, and T. Wischgoll, "Intuitive Error Space Exploration of Medical Image Data in Clinical Daily Routine," in EG/VGTC Conference on Visualization (EuroVis) Short Papers, 2017.

[25] N. Chamoli, S. Kukreja, and M. Semwal, "Survey and comparative analysis on entropy usage for several applications in computer vision," International Journal of Computer Applications, vol. 97, no. 16, 2014.

[26] U. Artmann, "Image quality assessment using the dead leaves target: experience with the latest approach and further investigations," in Digital Photography XI, vol. 9404. International Society for Optics and Photonics, 2015, p. 94040J.

[27] S. M. Pizer, E. P. Amburn, J. D. Austin, R. Cromartie, A. Geselowitz, T. Greer, B. ter Haar Romeny, J. B. Zimmerman, and K. Zuiderveld, "Adaptive histogram equalization and its variations," Computer vision, graphics, and image processing, vol. 39, no. 3, pp. 355-368, 1987. 
[28] J. R. Fienup, "Invariant error metrics for image reconstruction," Applied optics, vol. 36, no. 32, pp. 8352-8357, 1997.

[29] A. K. Boyat and B. K. Joshi, "A review paper: noise models in digital image processing," arXiv preprint arXiv:1505.03489, 2015.

[30] V. Jaya and R. Gopikakumari, "Iem: a new image enhancement metric for contrast and sharpness measurements," International Journal of Computer Applications, vol. 79, no. 9, 2013.

[31] Q. Tian, Q. Xue, N. Sebe, and T. S. Huang, "Error metric analysis and its applications," in Internet Multimedia Management Systems V, vol. 5601. International Society for Optics and Photonics, 2004, pp. 46-57.

[32] I. Irum, M. Sharif, M. Raza, and S. Mohsin, "A nonlinear hybrid filter for salt \& pepper noise removal from color images," Journal of Applied Research and Technology, vol. 13, no. 1, pp. 79 - 85, 2015.

[33] S. Cai, B. Gallina, D. Nyström, and C. Seceleanu, "Data aggregation processes: a survey, a taxonomy, and design guidelines," Computing, vol. 101, no. 10, pp. 1397-1429, 2019.

[34] C. Gillmann, "Image processing under uncertainty," doctoralthesis, Technische Universität Kaiserslautern, 2019. [Online]. Available: http://nbn-resolving.de/urn:nbn:de:hbz:386-kluedo-54707

[35] C. Gillmann, P. Arbelaez, J. Hernandez, H. Hagen, and T. Wischgoll, "An uncertainty-aware visual system for image pre-processing," Journal of Imaging, vol. 4, no. 9, p. 109, 2018.

[36] J.-L. Vay, A. Almgren, J. Bell, L. Ge, D. Grote, M. Hogan, O. Kononenko, R. Lehe, A. Myers, C. Ng et al., "Warp-x: A new exascale computing platform for beam-plasma simulations," Nuclear Instruments and Methods in Physics Research Section A: Accelerators, Spectrometers, Detectors and Associated Equipment, vol. 909, pp. 476479, 2018. 